\title{
Control de Internet: Las implicaciones políticas de las funciones de la IANA
}

\author{
Controlling Internet: Political Implications \\ of the IANA Functions
}

JELENA LAKETIC

Abogada independiente, Serbia

RESUMEN Este artículo tiene como finalidad describir los principales elementos que han posibilitado la supervisión de Internet por parte del gobierno estadounidense, lo que ha provocado importantes repercusiones institucionales y políticas. Se argumenta que el problema central del control de internet consistía tanto en la privatización fallida de la administración técnica, como la falta de legitimidad de la Internet Corporation for Assigned Names and Numbers (ICANN). Estas intervenciones del gobierno estadounidense pusieron en riesgo los derechos e intereses de distintos agentes, los cuales demandaban un mayor grado de participación en la administración de internet. La reciente transición de las facultades de la Internet Assigned Numbers Authority (IANA) del gobierno estadounidense a ICANN puede ser un avance real o simplemente constituir una estrategia para evitar el intervencionismo de otros Estados en la administración de Internet.

PALABRAS CLAVE Gobernanza de Internet, IANA, ICANN, nombres de dominio de internet.

ABSTRACT The article aims to describe how the United States govern- 
ment found itself in control of the Internet, which then led to important institutional and political repercussions. The article argues that the central issue of Internet control was the failed privatization of the technical management of the entire Internet root server system, and ICANN's lack of legitimacy. The United States government's intervention jeopardized the relevant rights and interests, which caused widespread protests from foreign states, demanding greater participation in the Internet governance issues. Within this context, the recent transition of IANA functions to ICANN can be a real progress in the governance of the Internet as we know it, or simply a rational strategy of the United States to avoid the intervention of other states in the administration of the Internet.

KEYWORDS Internet governance, IANA, ICANN, Internet domain names.

\section{INTRODUCCIÓN}

A pesar de que internet fue un proyecto financiado por el Departamento de Defensa de los Estados Unidos, lo que significa que originalmente no fue diseñado para uso masivo, su desarrollo como una red de interconexión mundial ha puesto en marcha una serie de efectos políticos sobre las estructuras sociales.

Dada la falta de una estructura formal en la gestión de internet, en un comienzo fragmentada dentro de diversas entidades bajo una compleja sucesión de relaciones contractuales entre estas entidades y el gobierno estadounidense, la falta de mecanismos de transparencia y rendición de cuenta se han convertido en la principal deficiencia en el control de internet.

A pesar de eso, internet logró imponerse en el uso diario puesto que la comunicación que se lleva a cabo en sus redes es significativamente diferente en comparación con otros tipos de intercambio de información, ya que este recurso se caracteriza por ser rápido, global y ofrecer inagotables opciones de cifrado.

Cuando a mediados de los años noventa el sistema comenzó a agrietarse, aumentó la conciencia sobre el valor económico de este recurso. Justo cuando se hacía evidente que internet poseía un enorme potencial económico, aparecieron las denominadas «malas prácticas». Dado que 
el aumento de la práctica de la ciberocupación o cybersquatting ${ }^{\mathrm{I}}$ amenazó los intereses económicos de importantes agentes a nivel global, los propietarios de marcas comerciales demandaban medidas eficaces por parte del Departamento de Comercio de los Estados Unidos de América. En I998, el gobierno estadounidense llamó a crear una nueva corporación que se encargase de la asignación de nombres de dominio, lo que resultaría en la conformación de la ICANN.

En este contexto comenzó el intervencionismo del gobierno estadounidense en la administración de internet cuyo fundamento se encuentra en el control que ejercía sobre las funciones de la IANA. En el período anterior a la creación de la ICANN, la IANA que era administrada por el Instituto de Ciencias de la Información de la Universidad del Sur de California ya supervisaba la coordinación de elementos claves que mantienen internet en funcionamiento. Estas funciones habitualmente se dividen en tres áreas: asignaciones de protocolos (IP), recursos numéricos de internet ${ }^{2}$ y gestión de la zona raíz. ${ }^{3}$

Este artículo plantea que los movimientos de la política interna de los Estados Unidos han transformado el régimen informal ad hoc de los inicios de la gestión de internet en un régimen más formal y centralizado a través de la creación de la ICANN.

I. Ciberocupación o cybersquatting o domain name grabbing consiste en el registro de un nombre de dominio de internet con el único propósito de venderlo posteriormente al titular legítimo de la marca por elevadas sumas de dinero. La ciberpiratería se refiere a las situaciones donde los ciberpiratas obtienen los nombres de dominio con el fin de desviar a los clientes desde el sitio del propietario de una marca a su sitio web para beneficiarse de clientes confundidos en cuanto a la procedencia de los productos o servicios ofrecidos.

2. La gestión de los recursos numéricos de internet implica la coordinación de los sistemas de direcciones del Protocolo de Internet a nivel global. No obstante, aparte de las direcciones de Protocolo de Internet (IP), los recursos numéricos de internet son también los números del Sistema Autónomo (AS o ASN), cuyo propósito es identificar de forma única un nombre de dominio de enrutamiento controlado por una administración única. En otras palabras, la administración de los recursos numéricos de internet proporciona la conectividad entre los operadores de la red.

3. La gestión de la zona raíz se refiere al nivel más alto del sistema jerárquico del sistema de los nombres de dominio de internet (DNS), lo que supone la asignación de operadores de los nombres de dominio de internet de nivel superior (TLD), incluyendo el mantenimiento técnico y administrativo, así como el registro de autorización de todos los nombres de dominio de internet de nivel superior (TLD). 
Asimismo se plantea que no sólo el propósito de la ICANN se determinó ex ante, en el sentido de que se estableció el marco contractual para consolidar la autoridad y el objetivo de este ente, sino la dinámica interna dentro de esta entidad refleja que detrás de un discurso constante de que internet puede funcionar únicamente como un medio abierto y libre se esconde la preferencia consciente de involucrar a actores no estatales en los arreglos pertinentes. Tal postura estadounidense y su dominio de este recurso global se han vuelto polémicas, incluso hasta el punto de fragmentar la red.

Teniendo en cuenta que la administración de internet estaba en un estado de emergencia por satisfacer las demandas crecientes de otras naciones, el I de octubre de 2016 finalizó oficialmente el contrato entre la ICANN y el gobierno estadounidense, de acuerdo con el proceso acordado en 1998 y postergado en varias ocasiones.

En este sentido, la reciente transición de custodia de las funciones de la IANA a la ICANN está íntimamente relacionada con potenciales riesgos y oportunidades. Aunque para muchos representa un momento histórico, pues se trata de la consecuencia lógica y esperada de la secuencia iniciada hace dos décadas, hay que destacar que fueron los constantes reclamos a través del tiempo los que han contribuido a que el gobierno estadounidense considere despojarse formalmente de su rol primordial en la gestión de internet. Sin embargo, disminuir su poder puede ser un movimiento estratégico bien pensado, ya que de esta manera se fortalecerán los intereses estadounidenses de limitar la intervención de las naciones extranjeras, conservando de cierto modo la posición primus inter pares en la toma de decisiones.

Está más que claro que con la expansión de internet, las crecientes demandas de compartir este valioso recurso no iban a disminuir. Bajo la creciente amenaza de lograr una regulación multilateral, el gobierno estadounidense optó por retirar su vínculo directo en relación a las funciones de la IANA a cambio de evitar la influencia que pueden ejercer distintos actores estatales, potenciando de esta forma el modelo multi-stakeholder. ${ }^{4}$

4. En un sentido más simple, el modelo multi-stakeholder o de múltiples partes interesadas se refiere a la participación en la toma de decisiones por parte de todos los actores que importan en un sistema. 
Para entender exactamente lo que implica la transición de las funciones de la IANA y las consecuencias que podría tener en la gobernanza de internet, es relevante la comprensión de dichas funciones dentro de un contexto histórico.

\section{ICANN}

El planteamiento de que el gobierno estadounidense se beneficia a través de una participación significativa en la gestión de internet no es ninguna novedad, ya que desde la creación de internet, Estados Unidos asumió el rol de liderazgo en su desarrollo. Desde un principio, el Departamento de Defensa de los Estados Unidos ejercía ciertos controles en relación con el sistema de los nombres de dominio de Internet (DNS).

Sabemos que los mecanismos y sistemas de comunicación interna invariablemente han sido muy importantes para los Estados, especialmente para las organizaciones militares. En este sentido, los inicios de internet están asociados con la formación de Arpanet, ${ }^{5}$ establecido por el gobierno estadounidense con el objetivo principal de potenciar la comunicación entre las instituciones militares y gubernamentales en la época de la Guerra Fría. Demostrando sus grandes beneficios en la facilitación de la interacción entre determinados grupos, Arpanet rápidamente logró extenderse a otros organismos gubernamentales, a los centros de educación superior y los demás servicios de investigación. «Como la red evolucionó mucho más allá de sus orígenes de investigación para incluir universidades, corporaciones y gente alrededor del mundo, Arpanet empezó a ser conocida como Darpa Internet y finalmente sólo Internet» (Abromson, 2004: 632).

Dado el ámbito en el cual se desarrolló internet, la red en un comienzo dependía de científicos e ingenieros informáticos. En el momento en que se evidenció que los nombres de dominio poseían un significativo valor comercial quedó demostrado que el sistema no estaba preparado para lidiar con las problemáticas legales.

Cabe decir que aunque todavía existe una percepción generalizada de

5. Arpanet (Advanced Research Projects Agency Network) fue una red de computadoras creada por encargo del Departamento de Defensa de los Estados Unidos con el objetivo de posibilitar la comunicación entre los distintos organismos gubernamentales. 
que la naturaleza de internet es de carácter descentralizada, en realidad las tres funciones básicas de internet son más bien centralizadas y se realizan en forma conjunta por una sola entidad, la ICANN. Estas funciones son las siguientes: a) el establecimiento de la política para la asignación de bloques de las direcciones en internet; b) el funcionamiento de los servidores raíz que hacen posible que los dispositivos de la red puedan encontrarse entre ellos y para que los paquetes de información puedan llegar de los remitentes a los receptores a través de la red; c) el establecimiento y cumplimento de las políticas para la creación y administración de dominios de nivel superior.

En consecuencia, la ICANN, formalmente una organización sin fines de lucro establecida en virtud del sistema jurídico de los Estados Unidos, desde su creación hasta el día de hoy siempre tuvo una posición clave en la gestión del sistema de los nombres de dominio. Dado que internet está jerárquicamente estructurada en una red de ordenadores que conecta redes más pequeñas, los nombres de dominio posibilitan que computadores conectados a internet puedan comunicarse correctamente entre sí. Por tanto, el valor de los nombres de dominio se encuentra en el hecho de que representan la condición insoslayable de la capacidad administrativa y técnica para tener visibilidad en la red.

El hecho de que las tres funciones específicas del direccionamiento estén centralizadas en una sola entidad ha sido fuertemente criticado en la literatura y se ha discutido si esta condición es una necesidad técnica o una decisión de diseño deliberada.

\section{¿CÓMO FUE POSIBLE CENTRALIZAR LAS FUNCIONES DE LA IANA DENTRO DE UNA SOLA ENTIDAD?}

Es importante destacar que la base del control que la ICANN tiene sobre el sistema DNS se estableció en un acuerdo entre dicha organización y el gobierno de los Estados Unidos (Memorandum of Understanding), el cual autoriza a la ICANN a realizar tareas específicas de carácter técnico relacionadas con la gestión de dicho sistema. En la literatura existe acuerdo de que otra condición sustancial para la gestión del sistema de los nombres de dominio por parte de la ICANN se encuentra en el hecho de que el gobierno estadounidense directamente apoyó a la ICANN a 
retomar la posición y las funciones de la IANA. ${ }^{6}$ En concordancia con lo planteado anteriormente, en el tiempo que precedió a la creación de la ICANN, el sistema de asignación de direcciones de internet ya había sido administrado por la IANA.

Uno de los pioneros en internet, John Postel, en base a un acuerdo con el gobierno estadounidense logró manejar la mayor parte de la administración de los nombres de dominio de internet dentro de la IANA. En I994, dicha entidad se convirtió en la máxima autoridad para la asignación de direcciones IP, la organización del sistema de los nombres de dominio, y muchos otros parámetros que se utilizan para apoyar a internet.

Por otra parte, en enero de I993, en nombre del gobierno de los Estados Unidos de América, la National Science Foundation (NSF) creó el InterNIC para la prestación de servicios relacionados con el registro de nombres de dominio de internet. Para poder cumplir con sus objetivos, el InterNIC contrató a tres empresas privadas para realizar funciones específicas. Éstas fueron: AT\&T, con el propósito de proporcionar los servicios de directorio, General Atomics, para proporcionar servicios de información, y Network Solutions, Inc. (NSI) para proporcionar el registro de nombres de dominio siguiendo las políticas establecidas por la IANA (Friedman y Siebert, I996: 635).

El logro más significativo de las medidas adoptadas por el gobierno estadunidense en aquel momento fue que la IANA transfirió algunas de sus competencias a la NSF, precisamente en su rama InterNIC. En la práctica esto significó que NSI realizaba tareas de registro, coordinación y mantenimiento del sistema de nombres de dominio en internet, registrando los nombres de dominio de nivel superior (TLD). Más importante aún, la NSI administraba el servidor raíz autoritativo, conocido como el servidor "A» que consiste en la base de datos que contiene los registros oficiales sobre registro de dominios efectuados mundialmente. Como consecuencia de estas circunstancias, a pesar de que AT\&T y General Atomics tenían funciones importantes, la NSI acaparaba todas las funciones clave.

Cabe decir que estos acontecimientos han sido blanco de diversas críticas. Se sostenía que el monopolio que poseía NSI no cumplía con

6. Véase, por ejemplo, Froomkin, 2000: 85-87 y Mueller, I999: 499-508. 
el principio de apertura de internet, tampoco con las normas de competencia. Al 30 de septiembre de I998, se habían registrado a través de NSI un total de 2.770 .000 nombres de dominio, lo que significó utilidades brutas por 35,9 millones de dólares estadounidenses para NSI, lo que motivó la presión de diversos agentes por compartir la administración de este valioso monopolio estadounidense (Schiavetta y Komaitis, 2003).

Además de la desaprobación por motivos económicos, las críticas de mayor importancia cuestionaban el arreglo político institucional de la regulación del sistema de los nombres de dominio de Internet. Respecto a esto, las mayores críticas recayeron en la cuestión de la legitimidad de la NSI debido a la relación directa que mantuvo con el gobierno estadounidense. En este contexto, incluso la Comisión Europea ha iniciado procedimientos contra este ente (Popovic y Besarovic, 2005: 8).

Como consecuencia de todo lo anterior, los gobiernos extranjeros buscaron una nueva gestión de internet para resaltar de una manera más apropiada las características globales de este valioso recurso.

Dentro de este marco social, la desaprobación generalizada respecto a la gobernanza de internet por parte de Estados Unidos, así como el crecimiento de las disputas sobre los nombres de dominio, y la falta de mecanismos especializados para combatir los registros y usos con mala fe, llevaron a que la administración de Clinton anunciara la intención de privatizar la gestión de internet, basándose en su valor comercial y en el aumento de la competencia. A través de la directiva titulada como «Marco para el Comercio Electrónico Mundial», del I de julio de I997, se establecieron los principios claves para guiar el apoyo del gobierno estadounidense en el desarrollo del comercio electrónico. Asimismo, se dieron instrucciones al Secretario de Comercio para privatizar el sistema DNS, tras la discusión sobre la futura administración de dicho sistema con un enfoque especial en los elementos globales y los asuntos relativos a los derechos de propiedad intelectual y las posibilidades de establecer un régimen de base contractual para la resolución de conflictos potenciales entre el uso de nombres de dominio y las marcas comerciales (Clinton y Gore, I997).

Cabe destacar que en el periodo de transición hacia un nuevo régimen de autorregulación, la autoridad principal para la administración de los nombres de dominio se transfirió de la NSF a la National Telecommuni- 
cations and Information Administration (NTIA), un organismo especializado del Departamento de Comercio. ${ }^{7}$

En enero de 1998 se expidió el Green Paper donde se elaboraron las propuestas de la NTIA, lo que impulsó intensos debates. Tomando en cuenta las críticas recibidas, el 3 de junio de 1998 se expidió el White Paper, donde se afirmó el propósito de establecer una entidad administradora de carácter privado sin fines de lucro, denominada «NewCo», con el propósito de armonizar las funciones específicas de los nombres de dominio de internet. En aquel momento, la NTIA estableció que NewCo debía ser una entidad con sede en el territorio estadounidense y una corporación sin fines de lucro.

A pesar de que dentro del White Paper el gobierno de los Estados Unidos sostenía que al establecer el NewCo se reconocería que internet es un medio global y que su gestión técnica debería reflejar plenamente la diversidad global de los usuarios, promoviendo la participación más activa de la comunidad de usuarios en las decisiones que afectan su gestión técnica, las críticas no cesaron. "Aunque la Comisión Europea respaldó el White Paper, éste fue fuertemente criticado por la Unión Europea, el Gobierno de Australia, y otros organismos que creían que el plan había habilitado a los Estados Unidos de América a retener demasiado control sobre el sistema DNS» (Proffitt, I999: 608).

No obstante, el I 8 de septiembre de I998 el gobierno de los Estados Unidos entregó la gestión del sistema de los nombres de dominio a la ICANN. De esta forma, reconoció implícitamente a la ICANN como el «NewCo» (Von Arx y Hagen, 2002: 7).

Es necesario indicar que todo lo anterior sugiere que desde la creación de la ICANN se requería implementar una serie de decisiones diseñadas para consolidar su autoridad. De especial relevancia es que todos estos movimientos suponían una característica común: la intervención del gobierno de los Estados Unidos.

Concretamente, la intervención del gobierno de los Estados Unidos para fortalecer la posición de la ICANN se reflejó en cuatro fundamentos contractuales:

7. NTIA es una agencia del Departamento de Comercio de los Estados Unidos y la principal responsable de asesorar al Presidente sobre cuestiones de política de información y telecomunicaciones. 
I. Los contratos entre el Departamento de Comercio de los Estados Unidos de América y la ICANN y los contratos entre el Departamento de Comercio y NSI/VeriSign donde se establece que NSI debe obtener la aprobación escrita del Departamento de Comercio para poder modificar el servidor raíz autoritativo. Fundamentalmente, esto se refiere a la facultad de añadir nuevos nombres de dominio de primer nivel.

2. Un Acuerdo de Investigación y Desarrollo en Colaboración (Cooperative Research and Development Agreement, CRADA) donde la ICANN se compromete a estudiar la función de la IANA en la fase experimental.

3. Un contrato sin licitación de adquisición de la función de la IANA.

4. El Memorando de Entendimiento entre el Departamento de Comercio y la ICANN que establece que la ICANN gestiona el sistema de los nombres de dominio en forma experimental, que prevé la cooperación entre las partes del acuerdo, aunque el Departamento de Comercio de los Estados Unidos de América retiene la supervisión final sobre el sistema (Von Arx y Hagen, 2002: Io).

Por consiguiente, está claro que la NTIA logró reunir a todas las partes con alguna participación relevante en la formulación de políticas dentro del sistema de DNS, con el propósito de consolidar la autoridad de la ICANN.

\section{¿QUÉ SIGNIFICARON LOS CONTRATOS MENCIONADOS CON RESPECTO A LAS FUNCIONES DE LA IANA?}

Principalmente, para poder ser reconocida como el «NewCo», como mencionamos antes, la ICANN tenía que asumir el papel de la IANA en la administración del sistema, dado que la IANA en virtud de un contratista independiente con el gobierno estadounidense ya estaba a cargo de las funciones que se propusieron privatizar. Con el propósito de posibilitar que la ICANN se haga cargo de estas funciones, el gobierno de los Estados Unidos apoyó directamente a la ICANN en base a un contrato en el cual autoriza a dicha entidad a estudiar la forma en que se pueden mejorar las funciones de la IANA. Es más, la sede de la ICANN se encuentra en Marina del Rey, en California, en el mismo edificio de oficinas que albergó a la IANA (Froomkin, 2000: 85).

Por tanto, el quid de la insuficiencia de lograr un mayor grado de in- 
clusión respecto a la gobernanza de Internet se encontraba en el poder de la ICANN de controlar unilateralmente el DNS a través de las funciones de la IANA.

Respecto a las funciones de la IANA es importante recalcar que la gestión de la zona raíz se polarizó en las funciones de formulación de políticas por un lado y funciones de carácter ejecutivo por el otro. Para ser más exactos, es importante señalar que a través del contrato entre el Departamento de Comercio y NSI/VeriSign se estableció la necesidad para NSI de obtener la aprobación escrita del Departamento de Comercio para poder modificar el servidor raíz autoritativo, reteniendo únicamente el control físico sobre el archivo de zona raíz. Por otro lado, este acuerdo ha posibilitado al gobierno estadounidense a ejercer un control sustancial sobre las operaciones de la ICANN. Debido a que las funciones de la IANA estaban siendo controladas por el gobierno estadounidense, era éste quien retenía el poder de presionar a la ICANN a cumplir con ciertas tareas al amenazar la transferencia de sus poderes a otra entidad, la cual actuaría como el «NewCo».

Esto es válido considerando el hecho de que la ICANN opera sobre la base de un Memorando de Entendimiento con el Departamento de Comercio de los Estados Unidos. Inclusive, dentro de la sección VII del Memorando de Entendimiento se prevé que el Departamento de Comercio de los Estados Unidos podrá rescindir el contrato con la ICANN mediante una notificación escrita dentro de un plazo de I 20 días, en cuyo caso todos los derechos contractuales de la ICANN se revertirían al gobierno estadounidense (SIMS y BURR, I999).

Más importante, ya que el contrato era por un período de tiempo limitado, la ICANN debía convertirse en un órgano totalmente privatizado e independiente después de un período de dos años, lo que no ocurrió en su debido momento, ya que la NTIA siempre aprovechaba la posibilidad de extender la relación contractual. ${ }^{8}$

8. La NTIA y la ICANN extendieron la relación contractual para el desempeño de las funciones de IANA en 200I, 2003 y 2006. Cuando el contrato entre el Departamento de Comercio y la ICANN expiró en el 2009, las partes negociaron un acuerdo bilateral en forma secreta constituyendo la Afirmación de Compromisos. El período base de desempeño para el contrato de $20 \mathrm{I} 2$ era del I de octubre de $20 \mathrm{I} 2$ hasta 30 de septiembre de 20I 5. Cuando se renovó el contrato entre la IANA y la ICANN, el 2 de julio de 20 I 2 , 
Por tanto, el hecho de que la posición de la ICANN siempre dependiera de la delegación de competencias del Departamento de Comercio de los Estados Unidos a través del Memorando de Entendimiento, sumado a la misma dinámica interna dentro del sistema de la gobernanza de Internet, permitieron que las naciones extranjeras vieran esto como una advertencia de los Estados Unidos por mantener su rol histórico en la autorización de cambios o modificaciones en el archivo raíz autoritativo.

Es significativo que esta postura de negarse a aceptar cualquier propuesta de transición, la cual supondría un resultado que incluiría un gobierno nacional o una organización intergubernamental, coincida con las preocupaciones que se han relevado en el Congreso estadounidense sobre la propuesta de transición de las funciones de la IANA, lo que se refería a las posibilidades de que la NTIA pierda la autoridad sobre la ICANN y el sistema DNS.

Todos estos sucesos han demostrado a la comunidad mundial que la reivindicación de la autoridad política final sobre la zona de la raíz era el aspecto más importante de la función del gobierno estadounidense en esta materia. Por esto, no son de extrañar las críticas sobre la posición de la ICANN, creada por el gobierno estadounidense, sujeta a la legislación estadounidense y cuya posición está determinada por la relación contractual con el gobierno de los Estados Unidos.

La existencia de pruebas inequívocas que sugerían que la ICANN permanecía sujeta a la intervención del gobierno estadounidense ha provocado una tormenta política y una gran desaprobación por parte del mundo académico.

\footnotetext{
se estableció expresamente que el contratista deberá ser una empresa de propiedad estadounidense y operada por una universidad estadounidense, que todas las operaciones y los sistemas primarios se mantendrán dentro de los Estados Unidos, y que el gobierno estadounidense reserva el derecho de inspeccionar los sistemas y procesos de todas las instalaciones y componentes utilizados para la ejecución del contrato. Si bien el contrato debía expirar el 30 de septiembre de 20 I 5 , el I 7 de agosto de 20 I 5 la NTIA anunció su intención de prorrogar dicha relación contractual, lo que significó que el contrato con la IANA se extendió por un año. Asimismo, la NTIA anunció su flexibilidad para extender el contrato por un período adicional hasta septiembre de 20 I9.
} 


\section{POR QUÉ SON IMPORTANTES LAS FUNCIONES DE LA IANA}

La gestión del sistema DNS tiene una importancia esencial, ya que este sistema sirve como vía para emparejar una secuencia numérica a un seudónimo fácil de recordar que sustituye a una irrecordable dirección de Protocolo de Internet. Como resume Froomkin:

El control sobre el DNS confiere poder sustancial a través de Internet. Quienquiera que controle el DNS decide qué nuevos tipos de nombres de dominio de nivel superior pueden existir (por ejemplo, nuevos sufijos como .xxx o .union) y cómo los nombres y los esenciales números de enrutamiento serán asignados a sitios web y otros recursos de Internet. Crear supone también el poder de destruir, y el poder de destruir trae consigo el poder de unir condiciones del uso de un nombre de dominio (Froomkin, 2000: 2I; la traducción es nuestra).

En esta línea es entendible que las políticas de Internet no sólo son objeto de una lucha en el sentido económico, ${ }^{9}$ sino que además la información representa una estructura crítica para alcanzar objetivos geopolíticos más amplios. El significado de las funciones de la IANA sugiere que la gestión de los nombres de dominio de Internet no se limita sólo a cuestiones técnicas, sino que abarca cuestiones legales, como los asuntos relativos a la propiedad intelectual e industrial, la libre expresión, el derecho a la sindicalización, las cuestiones de la privacidad, e incluso la identidad personal, nacional o regional.

Para ejemplificar esto, la autoridad administradora de estas funciones decide si un determinado nombre de dominio se puede agregar a la raíz y quién tiene derecho a operarlo. Asimismo, puede determinar que los registradores de nombres de dominio recojan los datos personales de los solicitantes de registro y que las prácticas de tratamiento de los datos recolectados puedan tener un impacto significativo, especialmente si se pueden hacer accesible al público.

9. Se estima que la IIoT (Industrial Internet of Things) podría contribuir a la economía mundial con I4,2 billones de dólares estadounidenses de la producción mundial en 2030. El término IIoT se refiere al concepto Internet Industrial de las Cosas que consiste en el uso de Internet de las Cosas (IoT) para interconectar las tecnologías de fabricación con internet. La internet de las cosas (IoT) es esencialmente un sistema de máquinas u objetos equipados con tecnologías de recopilación de datos, de manera que esos objetos pueden comunicarse entre sí. Véase Daugherty y Berthon (2015). 
En el caso de los nombres de dominio de nivel superior patrocinados (sponsored TLD), ${ }^{\text {,o }}$ se puede decidir no sólo si un determinado nombre de dominio se va a agregar a la raíz sino también qué comunidad específica va a estar a cargo del mismo.

Al negarse eventualmente a registrar un nombre de dominio internacionalizado (IDN) ${ }^{\mathrm{II}}$ a determinados usuarios de internet, se les puede negar la posibilidad de acceder a los nombres de dominio y los contenidos en su propio idioma. Ya en I996 Friedman y Siebert opinaron: «si el World Wide Web pretende ser verdaderamente global, debe no ser más anglocéntrica de lo que ya es. Un dominio .com o .biz no significa nada para un usuario que sólo habla japonés o árabe» (I996: 662).

En algunos casos, ciertas desviaciones de las reglas generales se transforman en asuntos políticos. A pesar de que los dominios de primer nivel de tipo geográfico, ccTLD (Country Code Top Level Domain), sirven como identificadores de los países, y para evitar el debate político y jurídico sobre qué entidad puede obtener su propio dominio nacional de primer nivel se adoptó la práctica de sincronizar los ccTLD con la lista ISO 3 I66-I, la situación especial del dominio .eu, demuestra que a la Unión Europea se le aprobó el registro de nombre de dominio geográfico, aunque la Unión Europa todavía no es un Estado. ${ }^{\mathrm{I2}}$ Por otro lado, el gTLD

Io. Un dominio de internet patrocinado (sTLD) es un dominio de nivel superior genérico especializado cuyo patrocinador representa a una comunidad específica, la cual establece y está a cargo de las responsabilidades delegadas para la formulación sobre diversos temas relacionados con el mismo. El registro bajo estos dominios se limita a miembros de las entidades específicas. Cada dominio patrocinado tiene su Reglamento. Las entidades que aspiran registrar un nombre de dominio patrocinado deben cumplir con ciertos requisitos. Por ejemplo, el dominio .museum por la Museum Domain Management Association, .post por la Universal Postal Union, .aero por la Société Internationale de Télécommunications Aéronautiques, entre otras.

I I. El nombre de dominio de internet internacionalizado (IDN) es de segundo nivel y contiene caracteres con acento diacrítico, como se requiere en muchos idiomas, incluyendo el castellano. Además, incluye los alfabetos de idiomas específicos, donde no se utiliza la escritura de alfabeto latino y es importante asegurar suficiente singularidad visual. Hoy en día es posible registrar los caracteres en el alfabeto cirílico, griego, armenio, hebreo, árabe, tailandés, tibetano, birmano, japonés, amárico (etíope), georgiano, chino, coreano, bengalí (Bangladesh), han tamil (Singapur), sinhala tamil (Sri Lanka), hindú, urdú, telugu, gujarati, punyabí, bengalí, tamil (India), entre otros.

I2. La lista ISO 3 I66-I está compilada por la Organización Internacional de Estan- 
.asia (para la región de Asia Pacifico) se estableció como un nombre genérico, lo que demuestra a priori que el vínculo entre la extensión y el territorio en algunos casos es débil, dado que los Estados asiáticos tienen la extensión .asia, la cual es de relevancia menor a la de la Unión Europea.

Además, en las regiones donde existen movimientos para establecer naciones independientes, la posibilidad de obtener un dominio de código de país se convierte en una lucha por obtener reconocimiento internacional. Es más, dado que las funciones de la IANA están centralizadas en una sola entidad, pueden impactar de manera más considerable en los usuarios a nivel mundial.

Considerando todo lo anterior, y sumando el hecho de que el servidor de archivos que contiene la lista de dominios de nivel superior se podía cambiar únicamente con el permiso del Departamento de Comercio de los Estados Unidos, ya que dentro del Memorando de Entendimiento se prevé que el Departamento de Comercio tiene la obligación de supervisar el funcionamiento del sistema de servidores raíz y las políticas relacionadas, a nivel práctico significó que el Departamento de Comercio tenía el poder de crear, modificar o eliminar los nombres de dominio de nivel superior (TLD), tanto los nombres de dominio de nivel superior genéricos (gTLD) como los nombres de dominio de nivel superior geográficos (ccTLD). Aunque en la época pre-ICANN, la IANA era responsable de proporcionar los servicios técnicos de coordinación a todos los usuarios de internet y se suponía que la ICANN bajo la intervención del gobierno estadounidense había retomado la posición de la IANA, la verdad es que la ICANN nunca fue autorizada para añadir, eliminar o modificar la información contenida en la zona raíz sin la autorización del gobierno estadounidense. Es más, este poder del gobierno estadounidense ya ha sido utilizado muchas veces. Sabemos que con el propósito de evitar el debate político y jurídico sobre qué entidad puede obtener su propio dominio nacional de primer nivel, estos se sincronizan con la lista ISO 3 I 66-I. En el caso de cambios en las fronteras estatales y, por tanto, si ocurren cambios de los nombres de determinados Estados, cuando la ISO realiza el cambio del código, la ICANN automáticamente hace lo mismo.

Sin embargo, en la doctrina se sostiene que el dominio de la Unión

darización (ISO) que proporciona códigos para los nombres de países. Así tenemos, por ejemplo, fr para Francia, it para Italia, .cl para Chile, .de para Alemania, entre otros. 
Soviética fue abolido mucho tiempo después de la eliminación del código de país «su» de la norma ISO, debido a las fuertes protestas suscitadas por dicha eliminación. En 2002, el nombre de dominio de nivel superior geográfico de Irak, que fue dirigido por una empresa estadounidense, fue suspendido por la ICANN debido al conflicto bélico, ya que el gobierno estadounidense argumentaba el legítimo derecho de proteger a su país del terrorismo (Von Arx y Hagen, 2002).

El caso más notorio en este sentido representa la creación del dominio .eu, así como el procedimiento de Resolución alternativa de controversias relativo a dicho nombre de dominio, el cual según muchos autores representa el producto de roces políticos entre la Unión Europea y los Estados Unidos. A pesar de que la Unión Europea se apresuró en establecer un adecuado marco normativo relativo al dominio .eu, la ICANN se demoró en delegarle la competencia exclusiva: «Por lo tanto, la Unión Europea puede regular, dirigir y crear tanto como quiera, pero la ICANN puede retrasar la 'creación' de eu a perpetuidad y con ello presionar a la Unión Europea en un acuerdo de patrocinio con arreglo a las especificaciones de la ICANN» (Von Arx y Hagen, 2002: Io).

En agosto de 2005 , la ICANN debía decidir sobre la aprobación del dominio .xxx de nivel superior exclusivo para los sitios de la industria del entretenimiento para adultos, pero la decisión se postergó en varias ocasiones, debido a la protesta formal por parte del gobierno del presidente Bush, que ejercía su poder de veto sobre el sistema de direcciones de internet. Es importante tener en cuenta que el aplazamiento de la aprobación del dominio .xxx únicamente por su naturaleza inmoral va más allá de un simple estándar técnico. El estándar técnico no supone la determinación si la elección de un determinado gTLD sirve mejor para poner en marcha determinadas políticas sociales y no cabe dentro de las facultades de la ICANN.

Por tanto, el gobierno de los Estados Unidos, a través del Departamento de Comercio, ejercía de facto el control sobre el sistema de los nombres de dominio. Algunos autores dejan claro que el propio gobierno estadounidense podría operar la raíz, pero no caben dudas de que el Departamento de Comercio estadounidense formalmente había cedido este control a la ICANN, para que esta corporación realizará las funciones que tal vez el gobierno estadounidense no estaba dispuesto de efectuar abiertamente (Froomkin, 200I: 605). 
Dentro de la estructura jerárquica del sistema de los nombres de dominio, el servidor principal es el servidor raíz, denominado como servidor «A». Este servidor actúa como la raíz principal controlando todos los aspectos relevantes del sistema, ya que contiene las direcciones IP de todos los servidores autorizados para dominios de nivel superior.

Según lo que indicó el Consejo Nacional de Investigación de los Estados Unidos, «el archivo zona raíz define al DNS. Para todos los efectos prácticos, un dominio de nivel superior (y, por lo tanto, todos sus dominios de nivel inferior) está en el DNS, y únicamente si aparece en el archivo zona raíz. Por lo tanto, la presencia en la raíz determina qué dominios están disponibles en internet» (National Research Council, 2005).

De acuerdo a ello, para poder ejercer un control sustancial sobre el sistema de los nombres de dominio, el control sobre el servidor raíz se convierte en conditio sine qua non. Este control equivale a un enorme poder económico y político, ya que quien controla el sistema de los nombres de dominio decide qué nuevos dominios se pueden añadir al servidor raíz, así como la forma en que se asignan. En teoría, siempre ha sido polémico el hecho de que el servidor raíz "A» esté bajo tutela de NSI, luego adquirida por VeriSign, ya que se trata de un monopolio desde los inicios de internet, lo que permitía conservar las dos funciones más relevantes en internet: ejercer el control sobre el servidor raíz autoritativo y gestionar la base de datos WhoIs. ${ }^{{ }^{3} 3}$ Por consiguiente, Estados Unidos siempre lograba mantener su control en la jerarquía de internet, ya que retenía la posesión física y jurídica sobre el servidor raíz «A» oponiéndose a compartir este valioso recurso incluso con la propia ICANN.

Teniendo en cuenta que este recurso se puede convertir en un medio para ejercer influencia extraterritorial sobre las naciones extranjeras, no es sorprendente que los gobiernos extranjeros siempre manifestaron su preocupación por la dominación estadounidense, la que veían como falta de una verdadera intención de internacionalizar la gestión de internet, demandando la igualdad de condiciones en cuestiones de políticas públicas internacionales relacionadas con internet.

I3. WhoIs es un protocolo TCP que proporciona información sobre los titulares y las aplicaciones de los nombres de dominio con el fin de garantizar la transparencia del sistema de nombres de estos hacia el público y para los fines de la administración de nombres de dominio. 
Del mismo modo, al involucrar en trámite al Departamento de Comercio, el gobierno estadounidense demostró que no estaba interesado en una política de privatización de internet, como se sostenía oficialmente.

Igualmente, es relevante que la influencia de la ICANN se expandió. Si bien la ICANN siempre ha sostenido que no tiene autoridad reglamentaria de ningún tipo, sino que únicamente actúa dentro de sus facultades, estableciendo las normas técnicas, tal como se estipula dentro del White Paper, la situación en la práctica difiere de tales declaraciones. La fijación de los modelos de negocio de los registradores, la acreditación de los proveedores de la solución de controversias, la creación de las normas sustantivas y del procedimiento para la resolución de controversias, tanto como la aplicación de los mismos, no representan tareas de carácter técnico (Froomkin, 2000: 96).

La preocupación respecto a estos temas con los años ha ido aumentando, teniendo en cuenta que internet como un medio de comunicación se ha convertido en un valioso recurso comercial con tendencia de esparcirse a diversos ámbitos de la realidad social.

Muchos gobiernos estimaron que la Organización de las Naciones Unidas, específicamente la Unión Internacional de Telecomunicaciones (UIT), como el organismo especializado para las Tecnologías de la Información y la Comunicación, se debería hacer responsable en gestionar internet. La comunidad internacional sostenía que internet era un nuevo medio de comunicación, por lo que su administración tenía que realizarse a través de los instrumentos tradicionales del derecho internacional. En 20I 2, la UIT convocó la Conferencia Mundial de Telecomunicaciones Internacionales (CMTI), y hasta parecía que la UIT podría lograr obtener cierto control, sin embargo, se mantuvo el statu quo.

Un cambio real en la dinámica política que regía estos temas se produjo en agosto de 2013 cuando Edward Snowden reveló la existencia de vigilancia de las comunicaciones de los usuarios a través de los servicios que usan a diario por parte de agencias gubernamentales, lo que provocó una ola de protestas. Sus filtraciones han tenido un efecto significativamente mayor en reducir el control estadounidense sobre internet que otras crisis políticas.

En marzo de 20I4, la administración del presidente Obama anunció la propuesta para entregar la supervisión de funciones de la IANA en el 
proceso de transición de las funciones claves de nombres de dominio de Internet a la comunidad multisectorial global (NTIA, 20I4).

Después de dos etapas que marcaron la evolución de la gobernanza de Internet, la primera la cual se caracteriza por la privatización fallida de la administración técnica de los servidores raíz, y la segunda la que tiene que ver con la falta de legitimidad de la ICANN, hoy en día se habla de un momento histórico de internet.

\section{LA TRANSICIÓN DE LAS FUNCIONES DE LA IANA}

Respecto a la reciente transición de las funciones de la IANA, podemos decir que representa la primera oportunidad de iniciar reformas sustanciales dentro del sistema DNS. Su propósito es eliminar la palanca contractual a través de la cual el gobierno estadounidense ha ejercido el control sobre la ICANN respecto a la gestión del sistema DNS.

Sin embargo, dicha transición se basa en la noción de que el sistema DNS debe ser manejado por un complejo sistema empleando el modelo multi-stakeholder. La característica clave es que este tipo de compartición de poder se lleva a cabo principalmente con la sociedad civil en sus múltiples formas, en lugar de otros Estados que pueden tener agendas muy diferentes que los Estados Unidos de América. Si hasta ahora el sistema ha sido influenciado por el gobierno estadounidense, no sólo porque internet se ha desarrollado en el territorio estadounidense, sino especialmente a través de asociaciones público-privadas que han resistido durante mucho tiempo a la posibilidad de mayor inclusión e internacionalización en la gestión del sistema DNS, la cuestión que nos debería alarmar es ¿quién va a ejercer la supervisión dentro de la ICANN y cómo se va a estructurar el sistema de rendición de cuentas?

Cabe decir que la independencia de la ICANN en los Estados Unidos es objeto de desaprobación de ciertos grupos de interés que insisten en que se permitirá a gobiernos de países autocráticos como China, Irán y Rusia a obtener un mayor grado de control sobre el contenido disponible en línea. Los republicanos plantean que la transición de las funciones de la IANA pone en peligro la libertad de expresión. Incluso, la transición de las funciones de la IANA se ha denunciado en el Congreso como una medida equivalente a la decisión del Presidente Jimmy Carter de transferir el Canal de Panamá, solo con consecuencias mucho peores (Zittrain, 20I4). 
Por otra parte, los así denominados países autocráticos tampoco se encuentran contentos. El director de la autoridad nacional para el registro de los nombres de dominio geográficos en Rusia, Andrei Vorobyov, destaca que esta transición es sólo el primer paso hacia la transferencia del control a través de Internet para la comunidad mundial. En particular, el experto señaló que la ICANN y la nueva estructura, que ejercerá las funciones de la IANA, todavía están sujetas a la jurisdicción de los tribunales estadounidenses, dado que la ICANN está situada en California y está sujeta a las leyes de dicho Estado. Andrei añade que si bien es difícil hacer pronósticos, es posible que el aumento de la independencia de segmentos individuales signifique que el rescate de ahogamiento se convierta en la obra de ahogamiento.

Tales reacciones no son extrañas, dado que hasta ahora la gobernanza de Internet siempre era dirigida por un número reducido de participantes, los cuales no tenían una intención real de poner en marcha un adecuado diseño institucional con un apropiado marco regulatorio e instrumentos de intervención administrativa que aumentarán el desarrollo tecnológico y económico sin expensas de mayores costos sociales.

La ICANN desde su inicio sufre de una falta de diseño interno constitucional. Dado que el gobierno estadounidense ejercía una importante influencia sobre la ICANN y el sistema de los nombres de dominio de internet, los elaboradores de políticas de gestión de internet «no trataron de forjar acuerdos sobre principios subyacentes y normas para la cooperación internacional sobre la gobernanza de internet, de manera que cuando trataron de crear normas y procedimientos globales, no existía consenso» (Mueller y otros, 2007: 238).

Todo esto puso en relieve el carácter atípico de la ICANN. Hasta se puede decir que a través de la intervención del gobierno estadounidense se ha establecido una estructura institucional innovadora. Como señala Froomkin, la ICANN actúa como el principal registro, teniendo una posición clave en la gestión del sistema, ya que cualquier entidad que desee ofrecer servicios de registro de nombres de dominio bajo gTLDs con acceso directo a los registros gTLDs deberá obtener una acreditación de la ICANN (Froomkin, 200I). Además del registro de un dominio específico de nivel superior, la ICANN celebra los contratos con los registradores. La ICANN puede promulgar políticas en relación con las disputas sobre los nombres de dominio de internet y obligar a los regis- 
trantes a someterse a las mismas, incluso cuando las terceras personas que no forman parte de la relación contractual con la ICANN lo estimen necesario. Esto es significativo dado que a través de la Política Uniforme la ICANN ha creado un sistema global que puede servir como una alternativa a la legislación nacional. Al estructurar funciones contrapuestas dentro de una misma entidad, se estableció un diseño que está fuera del orden clásico de captura regulatoria, así como de estándares de control que se ejercen en estos tipos de diseños, lo que ha provocado polémica.

Por tanto, una de las preguntas fundamentales en el nuevo orden tras la transferencia de las funciones de la IANA debería referirse a la reorganización de la ICANN en el sentido de lograr una delimitación precisa entre los poderes de dicha entidad, así como el establecimiento de mecanismos internos del control y rendición de las cuentas.

Sin embargo, la estructura de la ICANN es bastante compleja, dado que aparte de la Junta Directiva supone de organizaciones de apoyo, comités consultivos y una serie de subgrupos que representan a diversos intereses. $^{\mathrm{I} 4}$

En un entorno así, no se hace tan difícil para la ICANN desviarse de su compromiso de tomar las decisiones en base a consenso. Incluso al observar las características de la Política Uniforme de la ICANN uno se da cuenta que las características más importantes de este mecanismo de solución alternativa de conflictos se definen principalmente por los intereses del demandante. ${ }^{15}$ Los titulares de los nombres de dominio de internet resaltarían otros valores que para ellos son relevantes de priorizar dentro de este mecanismo. La carencia de justicia fundamental junto con las limitaciones de la libertad de expresión se consideran como las faltas más importantes. ${ }^{16}$ Los críticos más acérrimos de la Política Uniforme

I4. Para mayor compresión consultar la página https://www.icann.org/en/system/files/ files/management-org-o8sepi 5-en.pdf.

I 5. Politica Uniforme de la ICANN (con frecuencia denominada como la UDRP, Uniform Domain Name Dispute Resolution Policy) es el procedimiento alternativo obligatorio para la resolución de conflictos en materia de nombres de dominio de internet.

I6. Las violaciones a la libertad de expresión han generado medidas concretas en los países que se han visto vulnerados. En el caso de Francia, la Corte Constitucional afirmó el valor constitucional de los nombres de dominio, ya que concluyó que la asignación, la renovación, transferencia o proceso de cancelación de un nombre de dominio no sólo deben respetar los derechos de propiedad intelectual, sino también la libertad de ex- 
son los que opinan que dicha política pone a los titulares de marcas en una ventaja injusta sobre los titulares de los nombres de dominio de internet.

Esto significa que la ICANN está sujeta a la presión política que se pueda ejercer para filtrar determinados puntos de vista, involucrar a determinados participantes y desarrollar las formas de procesos de toma de decisiones, lo que puede tener consecuencias de largo alcance afectando a cientos de millones de usuarios. En este ejemplo concreto, al priorizar los intereses del lobby marcario e ignorar numerosas protestas en relación con este asunto, como consecuencia todavía no tenemos una base sólida para tratar todas las cuestiones emergentes respecto a las disputas sobre los nombres de dominio de una forma más íntegra.

\section{CONCLUSIONES}

Operar las funciones de la IANA es un medio y un fin en sí. A pesar de que el gobierno estadounidense ha resistido durante un largo periodo la posibilidad de compartir su control sobre el sistema, para muchos éste podría ser un momento para internacionalizar la gobernanza de internet.

Sin embargo, la pregunta es ¿qué significa internacionalizar internet? En este contexto, es importante saber que la ICANN solía presentarse como una organización dirigida por el sector privado incluso antes de adoptar en su retórica el modelo multi-stakeholder.

Los actores privados ya desempeñaron un papel importante en la gobernanza de internet, y muchos de ellos están radicados en los Estados Unidos. La asignación de los nombres de dominio se configura dentro de la ICANN, la cual sigue ubicada en el territorio estadounidense, los registradores de nombres de dominio basados en el territorio estadounidense retienen ganancias económicas, asimismo como muchos de los fabricantes de equipos de red, las empresas de informática y software.

Dado que el modelo de multi-stakeholder se realiza principalmente a través de la sociedad civil, en lugar de otros Estados que tienen diferentes perspectivas en comparación con la postura estadounidense, parece

presión y la libertad empresarial. Constitutional Council, decisión 20I0-45, QPC, 6 de octubre de 20Io, disponible http://www.conseilconstitutionnel.fr/conseilconstitutionnel/ root/bank_mm/anglais/en20I045qpc.pdf. 
conveniente para los Estados Unidos ceder el control sobre las funciones de la IANA. Es más, dado que la ICANN ahora desempeña un rol significativamente más importante y fue ésta la que elaboró la propuesta final aceptada por el gobierno estadounidense, es muy probable que esta entidad mantendrá su estado actual, lo que significa que legalmente estará sujeta al control total estadounidense, dado que está constituida bajo legislación estadounidense y domiciliada en los Estados Unidos.

Para algunos, el concepto de múltiples partes involucradas en la toma de decisiones genera muchas expectativas. Para otros se vincula con el propósito al que puede asignarse.

Una cosa es segura: el éxito del nuevo arreglo dependerá del nuevo marco conceptual que se desarrolle para garantizar la confianza de la comunidad global.

\section{REFERENCIAS}

Abromson, Henry M. (2004). "The Uniform Domain Name Dispute Resolution Policy: Will Alternative Dispute Resolution Succeed Where the Courts Have Not? A Proposed Solution To An Imperfect System». Rutgers Conflict Resolution Law Journal, I.

Bartlett, Robert (I999). "The Domain Name System: A Case Study of the Significance of Norms to Internet Governance». Harvard Law Review, I I2: I657-I664. Disponible en http://scholarship.law.berkeley.edu/cgi/viewcontent.cgi? article=3391\&context=facpubs.

Clinton, William J. y Albert Gore (I997). «Framework for global electronic commerce». Disponible https://clinton4.nara.gov/textonly/ WH/New/Commerce/read.html.

Daugherty, Paul y Bruno Berthon (2015). Winning with the Industrial Internet of Things: How to Accelerate the Journey to Productivity and Growth. Dublín: Accenture.

Friedman, Neal J. y Kevin Siebert (1996). "The Name Is Not Always the Same». Seattle University Law Review, 20 (3): 63 I.

Froomkin, A. Michael (2000). «Wrong Turn in Cyberspace: Using ICANN to Route around the APA and the Constitution». Duke Law Journal, 50 (I): 2I-96.

-. (200I). «Icann's Uniform Dispute Resolution Policy-Causes and (Partial) Cures». Brooklyn Law Review, 67 (3): 605. 
MAYer-Schönberger, Viktor y Malte Ziewitz (2006). «Jefferson rebuffed: the United States and the future of internet governance». Columbia Science and Technology Law Review, 8, I 88.

Mueller, Milton (I999). «ICANN and Internet governance: sorting through the debris of 'self-regulation'». Info, the Journal of Policy, Regulation and Strategy for Telecommunications, Information and Media, I(6): 497-520.

Mueller, Milton, John Mathiason y Hans Klein (2007). «The Internet and global governance: Principles and norms for a new regime». Global Governance: A Review of Multilateralism and International Organizations, I3 (2): 237-254.

National Research Council (2005). «Committee on Internet Navigation and the Domain Name System, Technical Alternatives and Policy Implications, Signposts in Cyberspace: The Domain Name System and Internet Navigation». National Academy Press, Washington, DC.

NTIA (20I4). «NTIA Announces Intent to Transition Key Internet Domain Name Function». Disponible en http://www.ntia.doc.gov/pressrelease/20 I 4/ntia-announces-intent-transition-key-internet-domainname-functions.

Popovic, Dušan y Vesna Besarovic (2005). «Imena internet domena i pravo intelektualne svojine». Institut za Uporedno Pravo.

Proffitt, Angela (I999). «Drop the Government, Keep the Law: New International Body for Domain Name Assignment Can Learn from United States Trademark Experience». Loyola of Los Angeles Entertainment Law Review, I9 (3), 60I.

Schiavetta, Susan y Konstatinos Komaitis (2003) «ICANN's Role in Controlling Information on the Internet». International Review of Law, Computers \& Technology, I7 (3): 267-284.

Sharrock, Lisa M. (200I). «The Future of Domain Name Dispute Resolution: Crafting Practical International Legal Solutions from Within the UDRP Framework». Duke Law Journal, 5 I (2): 8I 7-849.

Sims, Joe y J. Burr BeCKWiTh (I999). "Memorandum of understanding between the U.S. Department of Commerce and Internet Corporation for Assigned Names and Numbers». Disponible en https://www. icann.org/resources/unthemed-pages/icann-mou-I 998-I I-25-en.

Von ARx, Kim G. y Gregory R. Hagen (2002). «Sovereign Domains: 
A Declaration of Independence of ccTLDs from Foreign Control». Richmond Journal of Law \& Technology, 9, 4-8.

ZitTRAIN, Jonathan (20I4). «No Barack Obama Isn't Handing Control of the Internet Over to China». The New Republic, 224. Disponible en https://newrepublic.com/article/II7093/ us-withdraws-icann-why-its-no-big-deal.

\section{SOBRE LA AUTORA}

Jelena Laketic es abogada. Licenciada en Derecho por la Universidad de Novi Sad, Serbia. Graduate Certificate in International Contracts and $\mathrm{Bu}-$ siness Law por American University Washington College of Law y Magíster en Derecho con Mención en Contratación Comparada e Internacional, Universidad de Chile. Su correo electrónico es j.laketic@gmail.com.

Artículo recibido el 13 de octubre y aprobado el i 8 de noviembre de 2016 
